\title{
LINEAR STABILITY EFFECT OF DENSELY DISTRIBUTED POROUS MEDIUM AND CORIOLIS FORCE ON SORET DRIVEN FERROTHERMOHALINE CONVECTION
}

\author{
R. SEKAR* and D. MURUGAN \\ Department of Mathematics, Pondicherry Engineering College \\ Puducherry - 605 014, INDIA \\ E-mail: rsekar@pec.edu
}

\begin{abstract}
The effect of Coriolis force on the Soret driven ferrothermohaline convection in a densely packed porous medium has been studied. A linear stability analysis is carried out using normal mode technique. It is found that stationary convection is favorable for the Darcy model, therefore oscillatory instability is studied. A small thermal perturbation is applied to the basic state and linear stability analysis is used for which the normal mode technique is applied. It is found that the presence of a porous medium favours the onset of convection. The porous medium is assumed to be variable and the effect of the permeable parameter is to destabilize the system. The present work has been carried out both for oscillatory as well as stationary instabilities. The results are depicted graphically.
\end{abstract}

Key words: Coriolis force, Darcy model, Soret effect, ferrofluid, thermohaline convection.

\section{Introduction}

A ferrofluid (compound of Latin ferrum, meaning iron, and fluid) is a liquid which becomes strongly magnetized in the presence of a magnetic field. A ferrofluid is a combination of microscopic magnetic particles suspended in a liquid, usually a solvent or water. Ferrofluids are used in loudspeakers to cool the voice coil and reside in the air gap between the voice coil and the speaker's magnet.

Ferrofluids are colloidal liquids made of nanoscale ferromagnetic, or ferromagnetic, particles suspended in a carrier fluid-usually an organic solvent or water. Each tiny particle is thoroughly coated with a surfactant to inhibit clumping. Large ferromagnetic particles can be ripped out of the homogeneous colloidal mixture, forming a separate clump of magnetic dust when exposed to strong magnetic fields. The magnetic attraction of nanoparticles is weak enough so the surfactant's van der Waals repulsion is sufficient to prevent magnetic clumping or agglomeration. Ferrofluids usually do not retain magnetization in the absence of an externally applied field and thus are often classified as "superparamagnets" rather than ferromagnets.

The difference between ferrofluids and magnetorheological fluids (MR fluids) is the size of the particles. The particles in a ferrofluid primarily consist of nanoparticles which are suspended by Brownian motion and generally will not settle under normal conditions. MR fluid particles primarily consist of micrometer-scale particles which are too heavy for Brownian motion to keep them suspended, and thus will settle over time because of the inherent density difference between the particle and its carrier fluid. These two fluids have very different applications as a result.

In ferrofluids, magnetic and liquid states coexist. On the surface, they are deceptively simple systems: a homogeneous phase comprised of three constituents, namely the magnetic particles, surfactant, and the liquid carrier. However, a complex chemistry and a balance of inter-particle forces determine the colloidal stability. The magnetic particles must be in the range of $10 \mathrm{~nm}$. The surfactant tails should be long enough to prevent particle agglomeration. Ferrofluids synthesized about 30 years ago are still stable.

\footnotetext{
" To whom correspondence should be addressed
} 
Initially, Finlayson [1] studied the convective instability of a single component ferrofluid layer heated from below in the presence of a vertical uniform magnetic field and explained the concept of thermo mechanical interaction in a ferrofluid. Das Gupta and Gupta [2] showed the stabilizing effect of rotation on setting up of convective instability in ferrofluids. The stability of the magnetic fluid penetration through a porous medium in a high uniform magnetic field oblique to the interface is studied by Zahn and Rosensweig [3]. The effect of the magnetic field along the vertical axis on thermoconvective instability in a ferromagnetic fluid saturating a rotating porous medium with the Darcy model was studied by Sekar et al. [4]. The same effect for a Brinkman model was also studied by Sekar et al. [5]. The effects of rotation and anisotropy of a porous medium on ferroconvection was analyzed by Vaidyanathan et al. [6]. This was extended to a study on the effect of rotation on ferrothermohaline convection saturating a porous medium by Sekar et al. [7]. The effect of rotation on ferrothermohaline convection was analyzed and linear theory was used by Sekar et al. [8]. Soret-driven thermosolutal convection was studied by Hurle et al. [9]. Soret-driven convection in ferrofluids using a non-linear analysis was analyzed by Ryskin et al. [10].

Soret-driven ferro thermohaline convection was studied by Vaidyanathan et al. [11]. Shevtsova et al. [12] carried out a study on the onset of convection in Soret driven instability. The effect of Coriolis force on a Soret driven ferrothermohaline convective system was studied by Sekar et al. [13]. Following this, the same analysis in a medium of sparse particle suspension was analyzed by Vaidyanathan et al. [14]. The effect of rotation on double diffusive convection in a magnetized ferrofluid with an internal angular momentum was studied by Sunil et al. [15]. The effect of Coriolis force on thermal convection in a couple stress fluid saturated rotating rigid porous layer was studied by Shivakumara et al. [16]. Paras Ram et al. [1718] discussed the ferrofluid flow with magnetic field dependent viscosity due to a rotating disk with and without a porous medium. More recently, the presence and absence of an anisotropy porous medium on Soret driven ferrothermohaline convection have been investigated by Sekar et al. [19-21] using Brinkman and Darcy models. Also, with and without magnetic field dependent (MFD) viscosity on Soret driven ferrothermohaline convection in an anisotropic porous medium was studied by Sekar and Raju [22-23]. The effect of temperature dependent viscosity and Coriolis force on Soret driven ferrothermohaline convection in a porous medium and anisotropy effect was studied by Sekar et al. [24-25]. Most recently, the presence and absence of Coriolis force on Soret driven ferrothermohaline convection saturating a densely packed anisotropic porous medium was studied by Sekar et al. [26-27].

In the present investigation, the convection of Soret-driven ferro thermohaline instability of multicomponent fluid layer heated from below and salted from above in a densely packed porous medium with Coriolis force is analysed. Linear stability analysis is used. The conditions for the onset of stationary and oscillatory instabilities are obtained.

\section{Formulation of problem}

A horizontal layer of an incompressible Boussinesq ferromagnetic fluid of thickness ' $d$ ' saturating a densely packed porous medium with Coriolis force in the presence of a transverse applied magnetic field heated from below and salted from above is considered. The temperature and salinity at the bottom and top surfaces $z= \pm d / 2$ are $T_{0} \pm \Delta T / 2$ and $S_{0} \pm \Delta S / 2$, respectively. Both the boundaries are taken to be free and perfect conductors of heat and solute. The Soret effect on the temperature gradient is considered. Further, the whole system is assumed to rotate with a uniform constant angular velocity $\Omega=(0,0, \Omega)$ and the vertical direction is taken as the $z$ axis. The mathematical equations governing the above investigation are as follows:

The continuity equation for an incompressible fluid is

$$
\nabla \cdot \boldsymbol{q}=0
$$

The corresponding momentum equation is

$$
\rho_{o} \frac{D \boldsymbol{q}}{D t}=-\nabla p+\rho \boldsymbol{g}+\nabla \cdot(\boldsymbol{H B})+2 \rho_{o}(\boldsymbol{q} \times \boldsymbol{\Omega})+\frac{\rho_{o}}{2} \nabla\left(|\boldsymbol{\Omega} \times \boldsymbol{r}|^{2}\right)-\frac{\mu}{k} \boldsymbol{q} .
$$


The temperature equation for an incompressible ferrofluid is

$$
\left[\rho_{o} C_{V, H}-\mu_{o} \boldsymbol{H} \cdot\left(\frac{\partial \boldsymbol{M}}{\partial \boldsymbol{T}}\right)_{V, H}\right] \frac{d T}{d t}+\mu_{o} T\left(\frac{\partial \boldsymbol{M}}{\partial T}\right)_{V, H} \cdot \frac{d \boldsymbol{H}}{d t}=K_{l} \nabla^{2} T+\phi .
$$

The mass flux equation is given by

$$
\frac{D S}{D t}=K_{s} \nabla^{2} S+S_{T} \nabla^{2} T
$$

where $\rho_{o}, \boldsymbol{q}=(u, v, w), g=(0,0,-g), k, t, p, \mu, \boldsymbol{H}, \boldsymbol{B}, C_{V, H}, T, \boldsymbol{M}, K_{1}, S, K_{S}, \Omega=(0,0, \Omega), S_{T}$ and $\phi$ are the fluid density, velocity, acceleration due to gravity, permeability of the porous medium, time, pressure, coefficient of viscosity, magnetic field, magnetic induction, heat capacity at constant volume and magnetic field, temperature, magnetization, thermal conductivity, salinity, concentration diffusivity, angular velocity, Soret coefficient and viscous dissipation factor containing second-order terms in velocity, respectively.

Maxwell's equations are

$$
\nabla . \boldsymbol{B}=0, \quad \nabla \times \boldsymbol{H}=0 .
$$

Further, $\boldsymbol{B}, \boldsymbol{M}$ and $\boldsymbol{H}$ are related by

$$
\boldsymbol{B}=\mu_{0}(\boldsymbol{M}+\boldsymbol{H}) .
$$

Using Maxwell's equation for non-conducting fluids, one can assume that the magnetization is aligned with the magnetic field and depends on the magnitude of the magnetic field, temperature and salinity, so that

$$
\boldsymbol{M}=\frac{\boldsymbol{H}}{H} M(H, T, S)
$$

The magnetic equation of state is linearized about the magnetic field $H_{0}$, the average temperature $T_{0}$ and the average salinity $S_{0}$ and so

$$
M=M_{0}+\chi\left(H-H_{0}\right)-K\left(T-T_{0}\right)+K_{2}\left(S-S_{0}\right)
$$

where $\chi=(\partial M / \partial H)_{H_{0}, T_{0}}$ is the susceptibility, $K=-(\partial M / \partial T)_{H_{0}, T_{0}}$ is the pyromagnetic coefficient and $K_{2}=(\partial M / \partial S)_{H_{0}, S_{0}}$ is the salinity magnetic coefficient.

The density equation of state for a Boussinesq two-component fluid is

$$
\rho=\rho_{0}\left[1-\alpha_{t}\left(T-T_{0}\right)+\alpha_{S}\left(S-S_{0}\right)\right]
$$

where $\alpha_{t}$ is the thermal expansion coefficient and $\alpha_{s}$ is the solute analog of $\alpha_{t}$.

The basic state is assumed to be the quiescent state and taking the components of the magnetization and magnetic field in the basic state to be $\left[0,0, M_{0}(z)\right]$ and $\left[0,0, H_{0}(z)\right]$, the basic state quantities obtained are 


$$
\begin{aligned}
& \boldsymbol{q}=\boldsymbol{q}_{b}=0, \quad \mathrm{p}=\mathrm{p}_{b}(z), \quad \frac{\partial T}{\partial z}=-\beta_{t} \Rightarrow T_{b}=T_{0}-\beta_{t} z, \quad \frac{\partial S}{\partial z}=\beta_{S} \Rightarrow S_{b}=S_{0}+\beta_{s} z, \\
& \boldsymbol{H}_{b}(Z)=\left[H_{0}+\frac{\mathrm{K}\left(T_{b}-T_{0}\right)}{1+\chi}-\frac{K_{2}\left(S_{b}-S_{0}\right)}{1+\chi}\right] \hat{\boldsymbol{k}}, \quad \boldsymbol{M}_{b}(Z)=\left[M_{0}-\frac{\mathrm{K}\left(T_{b}-T_{0}\right)}{1+\chi}+\frac{K_{2}\left(S_{b}-S_{0}\right)}{1+\chi}\right] \hat{\boldsymbol{k}}
\end{aligned}
$$

where $\beta_{t}$ and $\beta_{S}$ are non-negative constants and $\hat{\boldsymbol{k}}=(0,0, I)$ is the unit vector along the vertical direction.

\section{Linear stability analysis}

The basic state is disturbed by a small thermal perturbation. Consider a perturbed state such that $\boldsymbol{q}=\boldsymbol{q}^{\prime}, \quad p=p_{b}(z)+p^{\prime}, \quad \mu=\mu_{b}(z)+\mu^{\prime}, \quad T=T_{b}(z)+T^{\prime}, \quad \boldsymbol{H}=\boldsymbol{H}_{b}(z)+\boldsymbol{H}^{\prime}, \quad \boldsymbol{M}=\boldsymbol{M}_{b}(z)+\boldsymbol{M}^{\prime} \quad$ where $\boldsymbol{q}^{\prime}, \mathrm{p}^{\prime}, \mu^{\prime}, T^{\prime}, \boldsymbol{H}^{\prime}$ and $\boldsymbol{M}^{\prime}$ are perturbed variables which are assumed to be small. The perturbed state temperature and solute are $T=T_{0}-\beta_{t} z+T^{\prime}$ and $S=S_{0}+\beta_{s} z+S^{\prime}$. Let the components of the perturbed magnetization and the magnetic field be $\left(M_{1}^{\prime}, M_{2}^{\prime}, M_{0}(z)+M_{3}^{\prime}\right)$ and $\left(H_{1}^{\prime}, H_{2}^{\prime}, H_{0}(z)+H_{3}^{\prime}\right)$, respectively.

$$
\begin{aligned}
& H_{i}^{\prime}+M_{i}^{\prime}=\left(1+\frac{M_{0}}{H_{0}}\right) H_{i}^{\prime} \quad(i=1,2), \\
& H_{3}^{\prime}+M_{3}^{\prime}=(1+\chi) H_{3}^{\prime}-K T^{\prime}+K_{2} S^{\prime}+S_{T} K T^{\prime} .
\end{aligned}
$$

Let $\left(B_{1}, B_{2}, B_{3}\right)$ denote the components of $\boldsymbol{B}$.

Using Eq.(2.6), one gets the result $B_{i}=\mu_{0}\left(M_{i}^{\prime}+H_{i}^{\prime}\right)$ and Eqs (3.1) and (3.2) give

$$
\begin{aligned}
& B_{i}=\mu_{0}\left(1+\frac{M_{0}}{H_{0}}\right) H_{i}^{\prime} \quad(i=1,2), \\
& B_{3}=\mu_{0}\left[(1+\chi) H_{3}^{\prime}-K T^{\prime}+K_{2} S^{\prime}+S_{\mathrm{T}} K T^{\prime}+M_{0}+H_{0}\right] .
\end{aligned}
$$

When the first equation of Eq.(2.5) is used in Eq.(2.1) and the resulting equation is linearized with $B_{i}$ $(i=1,2,3)$ given by Eq.(3.3) and Eq.(3.4), we obtain the following components

$$
\begin{gathered}
\rho_{0} \frac{\partial u}{\partial t}=-\frac{\partial p}{\partial x}+\mu_{0}\left(M_{0}+H_{0}\right) \frac{\partial H_{1}^{\prime}}{\partial z}+2 \rho_{0} \nu \Omega-\frac{\mu}{k} u, \\
\rho_{0} \frac{\partial v}{\partial t}=-\frac{\partial p}{\partial y}+\mu_{0}\left(M_{0}+H_{0}\right) \frac{\partial H_{2}^{\prime}}{\partial z}-2 \rho_{0} u \Omega-\frac{\mu}{k} v,
\end{gathered}
$$




$$
\begin{aligned}
& \rho_{0} \frac{\partial w}{\partial t}=-\frac{\partial p}{\partial z}+\mu_{0}\left(M_{0}+H_{0}\right) \frac{\partial H_{3}^{\prime}}{\partial z}-\mu_{0} H_{3}^{\prime} K \beta_{t}+\frac{\mu_{0} K^{2} \beta_{t} T^{\prime}}{1+\chi}\left(1-S_{\mathrm{T}}\right)+\mu_{0} H_{3}^{\prime} K_{2} \beta_{s}+ \\
& -\frac{\mu_{0} K K_{2} \beta_{s} T^{\prime}}{1+\chi}\left(1-S_{\mathrm{T}}\right)-\frac{\mu_{0} K K_{2} \beta_{t} S^{\prime}}{1+\chi}+\frac{\mu_{0} K_{2}^{2} \beta_{s} S^{\prime}}{1+\chi}+\rho_{0} g \alpha_{t} T^{\prime}-\rho_{0} g \alpha_{s} S^{\prime}-\frac{\mu}{k} w .
\end{aligned}
$$

Differentiating Eqs (3.5)-(3.7) with respect to $x, y$ and $z$ respectively and adding them the following equation is obtained upon using Eq.(2.1)

$$
\begin{aligned}
& \nabla^{2} p=\mu_{0}\left(M_{0}+H_{0}\right) \frac{\partial}{\partial z}\left(\nabla \cdot \boldsymbol{H}^{\prime}\right)+2 \rho_{0} \Omega \xi+\mu_{0} K_{2} \beta_{s} \frac{\partial H_{3}^{\prime}}{\partial z}-\mu_{0} K \beta_{t} \frac{\partial H_{3}^{\prime}}{\partial z}+\frac{\mu_{0} K^{2} \beta_{t}}{1+\chi}\left(1-S_{T}\right) \frac{\partial T^{\prime}}{\partial z}+ \\
& -\frac{\mu_{0} K K_{2} \beta_{s}}{1+\chi}\left(1-S_{T}\right) \frac{\partial T^{\prime}}{\partial z}+\frac{\mu_{0} K_{2}^{2} \beta_{s}}{1+\chi} \frac{\partial S^{\prime}}{\partial z}-\frac{\mu_{0} K K_{2} \beta_{t}}{1+\chi} \frac{\partial S^{\prime}}{\partial z}+\rho_{0} g \alpha_{t} \frac{\partial T^{\prime}}{\partial z}-\rho_{0} g \alpha_{s} \frac{\partial S^{\prime}}{\partial z}
\end{aligned}
$$

where $\boldsymbol{H}^{\prime}$ has the components $\left(H_{1}^{\prime}, H_{2}^{\prime}, H_{3}^{\prime}\right)$. From the second equation of (2.5), $\boldsymbol{H}^{\prime}=\nabla \phi$ where $\phi$ is a scalar potential. Elimination of $p$ from Eqs (3.7) and (3.8) then gives

$$
\begin{aligned}
& \rho_{0} \frac{\partial}{\partial t}\left(\nabla^{2} w\right)=\mu_{0} K_{2} \beta_{s} \nabla_{1}^{2} H_{3}^{\prime}-2 \rho_{0} \Omega \frac{\partial \xi}{\partial z}-\mu_{0} K \beta_{t} \nabla_{1}^{2} H_{3}^{\prime}+\frac{\mu_{0} K^{2} \beta_{t}}{1+\chi}\left(1-S_{T}\right) \nabla_{1}^{2} T^{\prime}+\rho_{0} g \alpha_{t} \nabla_{1}^{2} T^{\prime}+ \\
& -\frac{\mu_{0} K K_{2} \beta_{s}}{1+\chi}\left(1-S_{T}\right) \nabla_{1}^{2} T^{\prime}+\frac{\mu_{0} K_{2}^{2} \beta_{s}}{1+\chi} \nabla_{1}^{2} S^{\prime}-\frac{\mu_{0} K K_{2} \beta_{t}}{1+\chi} \nabla_{1}^{2} S^{\prime}-\rho_{0} g \alpha_{s} \nabla_{1}^{2} S^{\prime}-\frac{\mu}{k} \nabla^{2} w
\end{aligned}
$$

where $\nabla_{1}^{2}=\frac{\partial^{2}}{\partial x^{2}}+\frac{\partial^{2}}{\partial y^{2}}$ and $\nabla^{2}=\nabla_{1}^{2}+\frac{\partial^{2}}{\partial z^{2}}$

We now proceed to a normal mode analysis of the above stability problem. Let us take

$$
\begin{aligned}
& f(x, y, z, t)=f(z, t) e^{i\left(k_{x} x+k_{y} y\right)}, \quad \phi=\phi(z, t) e^{i\left(k_{x} x+k_{y} y\right)}, \quad w=w(z, t) e^{i\left(k_{x} x+k_{y} y\right)}, \\
& T^{\prime}=\theta(z, t) e^{i\left(k_{x} x+k_{y} y\right)}, \quad S^{\prime}=S(z, t) e^{i\left(k_{x} x+k_{y} y\right)},
\end{aligned}
$$

with the wave number $k_{0}^{2}=k_{x}^{2}+k_{y}^{2}$.

The vertical component of the momentum equation can be calculated as

$$
\begin{aligned}
& \left(\rho_{0} \frac{\partial}{\partial t}+\frac{\mu}{k}\right)\left(\frac{\partial^{2}}{\partial z^{2}}-k_{0}^{2}\right) w=\frac{\mu_{0} K \beta_{t}}{1+\chi}\left[(1+\chi) \frac{\partial \phi}{\partial z}-K \theta\left(1-S_{T}\right)\right] k_{0}^{2}-\rho_{0} g \alpha_{t} k_{0}^{2} \theta+ \\
& -2 \rho_{0} \Omega \frac{\partial \xi}{\partial z}+\rho_{0} g \alpha_{s} k_{0}^{2}+\frac{\mu_{0} K_{2} \beta_{s}}{1+\chi}\left[(1+\chi) \frac{\partial \phi}{\partial z}+K_{2} S\right] k_{0}^{2}-\frac{\mu_{0} K K_{2}}{1+\chi}\left[\beta_{s}\left(1-S_{T}\right) \theta-\beta_{t} S\right] k_{0}^{2},
\end{aligned}
$$




$$
\left(\rho_{0} \frac{\partial}{\partial t}+\frac{\mu}{k}\right) \xi=2 \rho_{0} \Omega \frac{\partial w}{\partial z}
$$

where $\xi$ is the $z$ - component of vorticity given by $\xi=\frac{\partial v}{\partial x}-\frac{\partial u}{\partial y}$.

The modified Fourier heat conduction equation is

$$
\rho_{0} C_{V, H} \frac{\partial \theta}{\partial t}-\mu_{0} K T_{0} \frac{\partial}{\partial t}\left(\frac{\partial \phi}{\partial z}\right)=K_{1}\left(\frac{\partial^{2}}{\partial z^{2}}-k_{0}^{2}\right) \theta+\left[\rho_{0} C \beta_{t}-\frac{\mu_{0} K^{2} T_{0}^{2} \beta_{t}}{1+\chi}+\frac{\mu_{0} K K_{2} T_{0} \beta_{s}}{1+\chi}\right] w
$$

where

$$
\rho_{0} C=\rho_{0} C_{V, H}+\mu_{0} K H_{0} .
$$

The salinity equation is

$$
\frac{\partial S}{\partial t}+\beta_{s} w=K_{s}\left(\frac{\partial^{2}}{\partial z^{2}}-k_{0}^{2}\right) S+S_{T}\left(\frac{\partial^{2}}{\partial z^{2}}-k_{0}^{2}\right) \theta .
$$

Using the analysis similar to Finlayson (1970), one gets

$$
(1+\chi) \frac{\partial^{2} \phi}{\partial z^{2}}-\left(1+\frac{M_{0}}{H_{0}}\right) k_{0}^{2} \phi-K \frac{\partial \theta}{\partial z}\left(1-S_{T}\right)+K_{2} \frac{\partial S}{\partial z}=0 .
$$

The non-dimensional numbers can be written using

$$
\begin{aligned}
& t^{*}=\frac{v t}{d^{2}}, \quad w^{*}=\frac{w d}{v}, \quad \mathrm{~T}^{*}=\left(\frac{K_{1} a R^{1 / 2}}{\rho_{0} C_{V, H} \beta_{t} v d}\right) \theta, \phi^{*}=\left(\frac{(1+\chi) K_{1} a R^{1 / 2}}{K \rho_{0} C_{V, H} \beta_{t} v d^{2}}\right) \phi, z^{*}=\frac{z}{d}, \\
& a=k_{0} d, \quad D=\frac{\partial}{\partial z^{*}}, \quad S^{*}=\left(\frac{K_{s} a R_{s}^{1 / 2}}{\rho_{0} C_{V, H} \beta_{s} v d}\right) S, \quad v=\frac{\mu}{\rho_{0}}, \xi^{*}=\frac{\xi d^{2}}{v}, k^{*}=\frac{k}{d^{2}} .
\end{aligned}
$$

Then Eqs (3.11) - (3.15) become

$$
\left.\begin{array}{l}
\left(\frac{\partial}{\partial t^{*}}+\frac{1}{k^{*}}\right)\left(D^{2}-a^{2}\right) w^{*}=a R^{1 / 2}\left[M_{1} D \phi^{*}-\left(1+M_{1}\left(1-S_{T}\right) T^{*}\right)\right]+a R^{1 / 2} M_{1} M_{5} D \phi^{*}+ \\
-a R^{1 / 2} M_{1} M_{5}\left(1-S_{T}\right) T^{*}+a R_{S}^{1 / 2}\left[1+M_{4}+\frac{M_{4}}{M_{5}}\right] S^{*}-\left(T_{a}\right)^{1 / 2} D \xi^{*} \\
\left(\frac{\partial}{\partial t^{*}}+\frac{1}{k^{*}}\right) \xi^{*}=\left(T_{a}\right)^{1 / 2} D w^{*} .
\end{array}\right\}
$$




$$
\begin{aligned}
& \operatorname{Pr}\left[\frac{\partial T^{*}}{\partial t^{*}}-M_{2} \frac{\partial}{\partial t^{*}}\left(D \phi^{*}\right)\right]=\left(D^{2}-a^{2}\right) T^{*}+a R^{1 / 2}\left(1-M_{2}-M_{2} M_{5}\right) w^{*}, \\
& \operatorname{Pr} \frac{\partial S^{*}}{\partial t^{*}}=\tau\left(D^{2}-a^{2}\right) S^{*}-a R_{S}^{1 / 2} M_{6} w^{*}+S_{T}\left(\frac{M_{5}}{M_{6}}\right)\left(\frac{R_{S}}{R}\right)^{1 / 2}\left(D^{2}-a^{2}\right) T^{*}, \\
& D^{2} \phi^{*}-M_{3} a^{2} \phi^{*}-\left(1-S_{T}\right) D T^{*}+\frac{M_{5}}{M_{6}}\left(\frac{R}{R_{S}}\right)^{1 / 2} D S^{*}=0
\end{aligned}
$$

where the non-dimensional parameters used are

$$
\begin{aligned}
& M_{1}=\frac{\mu_{0} K^{2} \beta_{t}}{(1+\chi) \rho_{0} g \alpha_{t}}, M_{2}=\frac{\mu_{0} K^{2} T_{0}}{(1+\chi) \rho_{0} C_{v, H}}, M_{3}=\frac{1+M_{0} / H_{0}}{(1+\chi)}, \\
& M_{4}=\frac{\mu_{0} K^{2} \beta_{s}}{(1+\chi) \rho_{0} g \alpha_{s}}, \quad M_{5}=\frac{K_{2} \beta_{s}}{K \beta_{t}}, M_{6}=\frac{K_{S}}{K_{1}}, \tau=\rho_{0} C_{v, H}\left(\frac{K_{S}}{K_{1}}\right), \\
& \operatorname{Pr}=\frac{\mu C_{v, H}}{K_{1}}, R_{S}=\frac{\rho_{0} C_{v, H} \beta_{s} \alpha_{s} g d^{4}}{v K_{S}}, \mathrm{R}=\frac{\rho_{0} C_{v, H} \beta_{t} \alpha_{t} g d^{4}}{v K_{l}},
\end{aligned}
$$

where $R_{S}$ is the salinity Rayleigh number, $\mathrm{R}$ is the thermal Rayleigh number, $\mathrm{Pr}$ is the Prandtl number and other parameters represent non-dimensional parameters used appropriately.

\section{Analysis of solution at free boundaries}

The boundary conditions on velocity, temperature and salinity are

$$
w^{*}=D^{2} w^{*}=T^{*}=D \phi^{*}=S^{*}=\xi^{*}=D \xi^{*}=0 \quad \text { at } \quad z^{*}= \pm 1 / 2 .
$$

The exact solutions satisfying Eq. (4.1) are

$$
\begin{aligned}
& w^{*}=A e^{\sigma t^{*}} \cos \pi z^{*}, \quad T^{*}=B e^{\sigma t^{*}} \cos \pi z^{*}, \quad S^{*}=C e^{\sigma t^{*}} \cos \pi z^{*}, \\
& D \phi^{*}=F e^{\sigma t^{*}} \cos \pi z^{*}, \quad \phi^{*}=\frac{F}{\pi} e^{\sigma t^{*}} \sin \pi z^{*}
\end{aligned}
$$

where $A, B, C$ and $F$ are constants. These functions substituted in the set of Eqs (3.16) - (3.20) give the following four linear homogeneous algebraic equations in $A, B, C$ and $F$. Removing the asterisks for our convenience, we get 


$$
\begin{aligned}
& {\left[\left(\sigma+\frac{1}{k}\right)\left(\pi^{2}+a^{2}\right)+\frac{T_{a} \pi^{2}}{\left(\sigma+\frac{l}{k}\right)}\right] A-a R^{1 / 2}\left[1+M_{1}\left(1-S_{T}\right)\left(1+M_{5}\right)\right] B+} \\
& +a R_{S}^{1 / 2}\left(1+M_{4}+M_{4} M_{5}^{-1}\right) C+a R^{1 / 2} M_{1}\left(1+M_{5}\right) F=0, \\
& a R^{1 / 2}\left[1-M_{2}\left(1+M_{5}\right)\right] A-\left(\pi^{2}+a^{2}+P_{r} \sigma\right) B+P_{r} \sigma M_{2} E=0, \\
& a R_{S}^{1 / 2} M_{6} A+S_{T}\left(\frac{M_{5}}{M_{6}}\right)\left(\frac{R_{S}}{R}\right)^{1 / 2}\left(\pi^{2}+a^{2}\right) B+\left[\tau\left(\pi^{2}+a^{2}\right)+\sigma P_{r}\right] C=0, \\
& R_{S}^{1 / 2} \pi^{2}\left(1-S_{T}\right) B-R^{1 / 2} \pi^{2}\left(\frac{M_{5}}{M_{6}}\right) C-R_{S}^{l / 2}\left(\pi^{2}+a^{2} M_{3}\right) E=0
\end{aligned}
$$

For the existence of non-trivial eigen functions, the determinant of the coefficients of $A, B, C$ and $F$ in Eqs (4.3) - (4.6) must vanish. Following the techniques and analysis of Sekar et al. (2007), Eqs (4.3)-(4.6) leads to

$$
U \sigma^{4}+V \sigma^{3}+W \sigma^{2}+X \sigma+Y=0
$$

where

$$
\begin{aligned}
& U=\left(\pi^{2}+a^{2}\right)\left(\pi^{2}+a^{2} M_{3}\right) P_{r}^{2}, \\
& V=\left(\pi^{2}+a^{2} M_{3}\right)\left[\left(\pi^{2}+a^{2}\right)^{2}(1+\tau)+\frac{2}{k}\left(\pi^{2}+a^{2}\right) P_{r}\right] P_{r} \\
& W=\left(\pi^{2}+a^{2} M_{3}\right)\left(\pi^{2}+a^{2}\right)\left[\tau\left(\pi^{2}+a^{2}\right)^{2}+\frac{2}{k}\left(\pi^{2}+a^{2}\right) P_{r}(1+\tau)\right]+ \\
& +a^{2} R \mathrm{P}_{r} M_{1}\left(1+M_{5}\right)\left[\left(1-S_{\mathrm{T}}\right)\left(\pi^{2}+a^{2} M_{3}\right)-\pi^{2}\left(1-S_{\mathrm{T}}+M_{5}\right)\right]+a^{2} R \mathrm{P}_{r}\left(\pi^{2}+a^{2} M_{3}\right)+ \\
& +a^{2} R_{S} P_{r}\left(\pi^{2}+a^{2} M_{3}\right)\left(1+M_{4}+M_{4} M_{5}^{-1}\right) M_{6}+\left(\pi^{2}+a^{2} M_{3}\right)\left[T_{a} \pi^{2}+\frac{1}{k^{2}}\left(\pi^{2}+a^{2}\right)\right] P_{r}^{2}, \\
& X=\left(\pi^{2}+a^{2} M_{3}\right)\left(\pi^{2}+a^{2}\right)\left[\frac{1}{k^{2}}(1+\tau)\left(\pi^{2}+a^{2}\right) P_{r}+\pi^{2} T_{a} \mathrm{P}_{r}+\frac{2}{k} \tau\left(\pi^{2}+a^{2}\right)^{2}\right]+ \\
& +a^{2} R \tau\left(\pi^{2}+a^{2} M_{3}\right)\left(\pi^{2}+a^{2}\right)\left[1+\left(1-S_{\mathrm{T}}\right) M_{1}\left(1+M_{5}\right)\right]+ \\
& +\frac{1}{k} a^{2} R \mathrm{P}_{r}\left[\left(\pi^{2}+a^{2} M_{3}\right)\left\{1+M_{1}\left(1+M_{5}\right)\left(1-S_{\mathrm{T}}\right)\right\}-\pi^{2} M_{1}\left(1+M_{5}\right)\left\{\left(1-S_{\mathrm{T}}\right)+M_{5}\right\}\right]+ \\
& -a^{2} R\left(\pi^{2}+a^{2}\right) M_{1}\left(1+M_{5}\right) \pi^{2}\left[S_{\mathrm{T}}\left(\frac{M_{5}}{M_{6}}\right)^{2}+\tau\left(1-S_{\mathrm{T}}\right)+M_{5}\right]+ \\
& +a^{2} R_{s}\left(\pi^{2}+a^{2} M_{3}\right)\left(1+M_{4}+M_{4} M_{5}^{-1}\right)\left[\frac{1}{k} M_{6} P_{r}+\left(\pi^{2}+a^{2}\right)\left\{S_{\mathrm{T}}\left(\frac{M_{5}}{M_{6}}\right)+M_{6}\right\}\right],
\end{aligned}
$$




$$
\begin{aligned}
& Y=\tau\left(\pi^{2}+a^{2} M_{3}\right)\left(\pi^{2}+a^{2}\right)^{2}\left[T_{a} \pi^{2}+\frac{1}{k^{2}}\left(\pi^{2}+a^{2}\right)\right]+ \\
& +\frac{1}{k} a^{2} R \tau\left(\pi^{2}+a^{2} M_{3}\right)\left(\pi^{2}+a^{2}\right)\left[1+\left(1-S_{\mathrm{T}}\right) M_{1}\left(1+M_{5}\right)\right]+ \\
& -\frac{1}{k} a^{2} R\left(\pi^{2}+a^{2}\right) M_{1}\left(1+M_{5}\right) \pi^{2}\left[S_{\mathrm{T}}\left(\frac{M_{5}}{M_{6}}\right)^{2}+\tau\left(1-S_{\mathrm{T}}\right)+M_{5}\right]+ \\
& +\frac{1}{k} a^{2} R_{S}\left(\pi^{2}+a^{2} M_{3}\right)\left(\pi^{2}+a^{2}\right)\left(1+M_{4}+M_{4} M_{5}^{-1}\right)\left[S_{\mathrm{T}}\left(\frac{M_{5}}{M_{6}}\right)+M_{6}\right] .
\end{aligned}
$$

\section{Stationary convection}

For obtaining stationary instability, the time-independent term $Y=0$. Equation (4.7) helps one to obtain eigen value $R^{s c}$ for which a solution exists

$$
R^{s c}=\frac{N_{r}}{D_{r}}
$$

where

$$
N_{r}=\left(\pi^{2}+a^{2}\right)\left[T_{a} \pi^{2} k+\frac{1}{k}\left(\pi^{2}+a^{2}\right)\right]-a^{2} R_{s} \tau^{-1}\left(1+M_{4}+M_{4} M_{5}^{-1}\right)\left[S_{\mathrm{T}}\left(\frac{M_{5}}{M_{6}}\right)+M_{6}\right]
$$

and

$$
D_{r}=a^{2}\left[1+\left(1-S_{\mathrm{T}}\right) M_{1}\left(1+M_{5}\right)\right]-\pi^{2}\left[\frac{a^{2} M_{1}\left(1+M_{5}\right)}{\pi^{2}+a^{2} M_{3}}\right]\left[S_{\mathrm{T}}\left(\frac{M_{5}}{M_{6}}\right)^{2} \tau^{-1}+\left(1-S_{\mathrm{T}}\right)+M_{5} \tau^{-1}\right]
$$

For very large $M_{l}$, one gets the results for the magnetic mechanism and the critical thermo magnetic Rayleigh number for stationary mode is obtained using

$$
N^{s c}=R^{s c} M_{1}=\frac{N_{r}}{D_{r}}
$$

where

$$
\mathrm{N}_{r}=\left(\pi^{2}+a^{2}\right)\left[T_{a} \pi^{2} k+\frac{1}{k}\left(\pi^{2}+a^{2}\right)\right]-a^{2} R_{s} \tau^{-1}\left(1+M_{4}+M_{4} M_{5}^{-1}\right)\left[S_{\mathrm{T}}\left(\frac{M_{5}}{M_{6}}\right)+M_{6}\right]
$$

and

$$
D_{r}=a^{2}\left[\left(1-S_{\mathrm{T}}\right)\left(1+M_{5}\right)\right]-\pi^{2}\left[\frac{a^{2}\left(1+M_{5}\right)}{\pi^{2}+a^{2} M_{3}}\right]\left[S_{\mathrm{T}}\left(\frac{M_{5}}{M_{6}}\right)^{2} \tau^{-1}+\left(1-S_{\mathrm{T}}\right)+M_{5} \tau^{-1}\right]
$$

\section{Oscillatory convection}

The conditions for the onset of oscillatory stabilities are obtained as follows. Taking $\sigma=i \sigma_{1}$ and $\sigma_{1}^{2}>0$, following the analysis and techniques of Sekar et al. (2007), the critical Rayleigh number for oscillatory mode has been calculated using

$$
R^{o c}=\frac{C_{2} A_{2}+B_{2} D_{2}}{A_{2}^{2}+B_{2}^{2}}
$$


where

$$
\begin{aligned}
& A_{2}=-U_{1} \sigma_{1}^{2}+V_{1}, \quad B_{2}=W_{1} \sigma_{1}, \quad C_{2}=-U_{2} \sigma_{1}^{4}+W_{2} \sigma_{1}^{2}-Y_{1}, \\
& D_{2}=V_{2} \sigma_{1}^{3}-X_{1} \sigma_{1}, \quad \sigma_{1}^{2}=\frac{-B_{1} \pm s q r t\left(B_{1}^{2}-4 A_{1} C_{1}\right)}{2 A_{1}} \\
& A_{1}=U_{2} W_{1}-U_{1} V_{2}, \quad B_{1}=V_{1} V_{2}+U_{1} X_{1}-W_{1} W_{2}, \quad C_{1}=W_{1} Y_{1}-V_{1} X_{1}, \\
& U_{1}=a^{2} P_{r} M_{1}\left(1+M_{5}\right)\left[\left(1-S_{\mathrm{T}}\right)\left(\pi^{2}+a^{2} M_{3}\right)-\pi^{2}\left(1-S_{\mathrm{T}}+M_{5}\right)\right]+a^{2} R P_{r}\left(\pi^{2}+a^{2} M_{3}\right) \\
& V_{l}=\frac{1}{k} a^{2} \tau\left(\pi^{2}+a^{2} M_{3}\right)\left(\pi^{2}+a^{2}\right)\left[1+\left(1-S_{\mathrm{T}}\right) M_{1}\left(1+M_{5}\right)\right]+ \\
& -\frac{1}{k} a^{2}\left(\pi^{2}+a^{2}\right) M_{1}\left(1+M_{5}\right) \pi^{2}\left[S_{\mathrm{T}}\left(\frac{M_{5}}{M_{6}}\right)^{2}+\tau\left(1-S_{\mathrm{T}}\right)+M_{5}\right] \\
& W_{1}=a^{2} \tau\left(\pi^{2}+a^{2} M_{3}\right)\left(\pi^{2}+a^{2}\right)\left[1+\left(1-S_{\mathrm{T}}\right) M_{1}\left(1+M_{5}\right)\right]+ \\
& +\frac{1}{k} a^{2} P_{r}\left[\left(\pi^{2}+a^{2} M_{3}\right)\left\{1+M_{1}\left(1+M_{5}\right)\left(1-S_{\mathrm{T}}\right)\right\}-\pi^{2} M_{1}\left(1+M_{5}\right)\left\{\left(1-S_{\mathrm{T}}\right)+M_{5}\right\}\right]+ \\
& -a^{2}\left(\pi^{2}+a^{2}\right) M_{1}\left(1+M_{5}\right) \pi^{2}\left[S_{\mathrm{T}}\left(\frac{M_{5}}{M_{6}}\right)^{2}+\tau\left(1-S_{\mathrm{T}}\right)+M_{5}\right] \\
& \mathrm{X}_{1}=\left(\pi^{2}+a^{2} M_{3}\right)\left(\pi^{2}+a^{2}\right)\left[\frac{1}{k^{2}}(1+\tau)\left(\pi^{2}+a^{2}\right) P_{r}+\pi^{2} T_{a} P_{r}+\frac{2}{k} \tau\left(\pi^{2}+a^{2}\right)^{2}\right]+ \\
& +a^{2} R_{s}\left(\pi^{2}+a^{2} M_{3}\right)\left(1+M_{4}+M_{4} M_{5}^{-1}\right)\left[\frac{1}{k} M_{6} P_{r}+\left(\pi^{2}+a^{2}\right)\left\{S_{\mathrm{T}}\left(\frac{M_{5}}{M_{6}}\right)+M_{6}\right\}\right] \text {, } \\
& Y_{1}=\tau\left(\pi^{2}+a^{2} M_{3}\right)\left(\pi^{2}+a^{2}\right)^{2}\left[T_{a} \pi^{2}+\frac{1}{k^{2}}\left(\pi^{2}+a^{2}\right)\right]+ \\
& +\frac{1}{k} a^{2} R_{s}\left(\pi^{2}+a^{2} M_{3}\right)\left(\pi^{2}+a^{2}\right)\left(1+M_{4}+M_{4} M_{5}^{-1}\right)\left[S_{\mathrm{T}}\left(\frac{M_{5}}{M_{6}}\right)+M_{6}\right], \\
& U_{2}=\left(\pi^{2}+a^{2}\right)\left(\pi^{2}+a^{2} M_{3}\right) P_{r}^{2}, \\
& V_{2}=\left(\pi^{2}+a^{2} M_{3}\right)\left[\left(\pi^{2}+a^{2}\right)^{2}(1+\tau)+\frac{2}{k}\left(\pi^{2}+a^{2}\right) P_{r}\right] P_{r} \\
& W_{2}=\left(\pi^{2}+a^{2} M_{3}\right)\left(\pi^{2}+a^{2}\right)\left[\tau\left(\pi^{2}+a^{2}\right)^{2}+\frac{2}{k}\left(\pi^{2}+a^{2}\right)(1+\tau) P_{r}\right]+ \\
& +a^{2} R_{S} P_{r}\left(\pi^{2}+a^{2} M_{3}\right)\left(1+M_{4}+M_{4} M_{5}^{-1}\right) M_{6}+\left(\pi^{2}+a^{2} M_{3}\right)\left[T_{a} \pi^{2}+\frac{1}{k^{2}}\left(\pi^{2}+a^{2}\right)\right] P_{r}^{2} .
\end{aligned}
$$




\section{Method of solution}

The Soret-driven thermoconvective instability of a ferromagnetic fluid heated from below and salted from above rotating in a densely packed porous medium has been analyzed using the Darcy model. The perturbation method is applied and a normal mode analysis is adopted. In the perturbation method, due to the application of a magnetic field, the system is perturbed from the basic state (quiescent state). Accordingly the governing and other equations are modified. Linear stability analysis is considered. Then normal mode analysis is taken. Non-dimensional analysis is carried out and the exact solutions satisfying the appropriate boundary conditions are taken yielding algebraic equations. For getting a non-trivial solution for the system of linear homogeneous equations, the coefficients of the dynamic variables are equated to zero and on simplification, the expression for $R^{s c}$ is obtained. Varying the values of the parameters in the allowable range and getting the corresponding $R^{s c}$ values, we get the stability pattern.

\section{Results and discussions}

Before discussing the significant results of the convective system, we turn our attention to the possible range of values of various parameters arising in the study. The Prandtl number Pr is assumed to be 0.01. The Taylor number Ta is assumed to vary from 10 to $10^{8}$. The Soret parameter $\mathrm{S}_{\mathrm{T}}$ is assumed to take values from -0.002 to 0.002 , the salinity Rayleigh number Ra takes values $-100,0,100,400$ and 600 . The non-buoyancy magnetization parameter $M_{3}$ is allowed to take values from 5 to 25 . The values of the ratio of mass transport to heat transport $\tau$ is assumed to be $0.03,0.05,0.07,0.09$ and 0.11 . The buoyancy magnetization parameter $M_{1}$ is assumed to be 1000. For these fluids, $M_{2}$ will have a negligible value and hence is taken as zero. $M_{6}$ is taken to be 0.1 and $M_{4}$ is the effect on magnetization due to salinity. This is allowed to vary from 0.1 to 0.5 taking values less than the non-buoyancy magnetization parameter $M_{3} . M_{5}$ represents the ratio of the salinity effect on the magnetic field and pyromagnetic coefficient. This is varied between 0.1 and 0.5 . The permeability of the porous medium $k$ is assumed to take the values from 0.001 , $0.003,0.005,0.007$ and 0.009 (Darcy number).

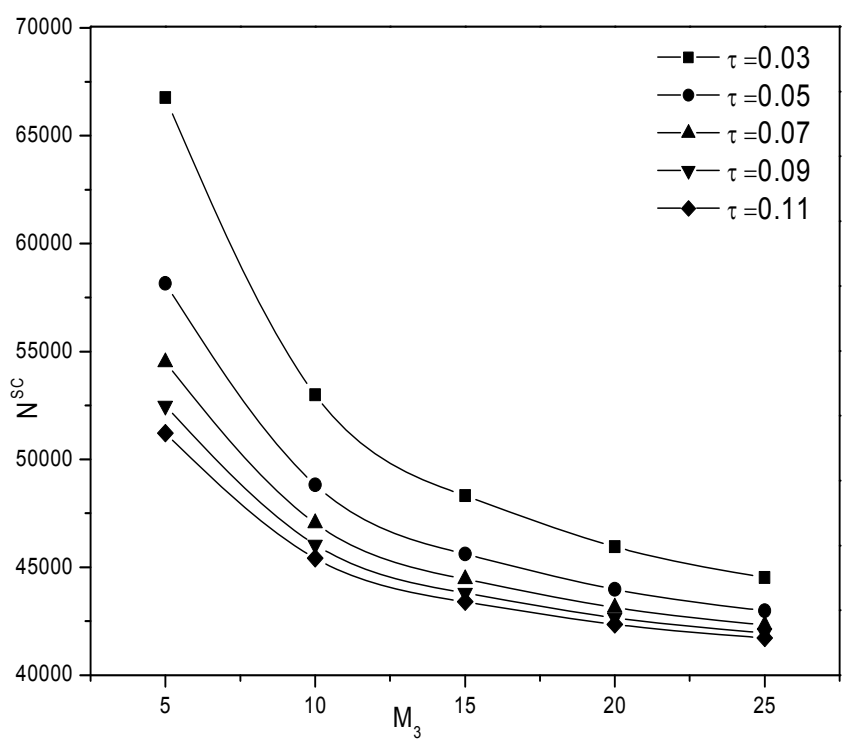

Fig.1. Variation of $N^{\mathrm{sc}}$ versus $M_{3}$ for different $\tau$ with $\mathrm{St}=-0.002, R_{S}=100, k=0.001$ and $\mathrm{Ta}=10^{5}$.

Figure 1 illustrates the variation of the thermal Rayleigh number $N^{s c}$ versus the non-buoyancy magnetization parameter $M_{3}$ for $\mathrm{Ta}=10^{5}$. When the values of the ratio of mass transport to heat transport $\tau$ is varied from 0.03 to 0.11 , it is seen that, when $M_{3}$ increases from 5 to $25, N^{\mathrm{sc}}$ decreases indicating the onset of 
instability. This is because high magnetization tends to release large energy to the system causing instability to set in earlier. Also, as the ratio of mass transport to heat transport $\tau$ increases from 0.03 to 0.11 , there is a fall in the values of $N^{\mathrm{sc}}$. Thus larger values of $\tau$ lead to destabilization of the system. The magnetization of the fluid is found to destabilize the system through oscillatory mode, which was discussed by Sekar et al. (2007).

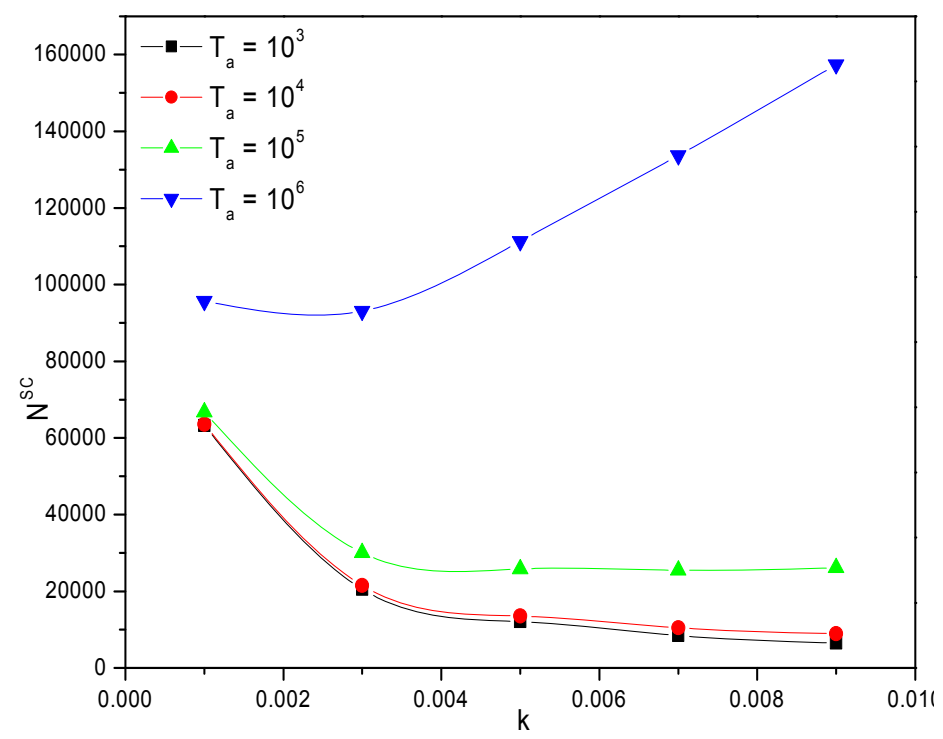

Fig.2. Variation of $N^{\mathrm{sc}}$ versus $k$ for different Ta with $\mathrm{St}=-0.002, R_{S}=-500, \tau=0.03$ and $M_{3}=5$.

Figure 2 shows the variation of the thermal Rayleigh number $N^{\text {sc }}$ versus the permeability of the porous medium $k$. It has been observed that as the Taylor number Ta is increased from $10^{3}$ to $10^{6}$, it is clear that as the permeability $k$ increases from 0.001 to 0.009 , the thermal Rayleigh number $N^{\text {sc }}$ values tend to decrease for small values of Ta, whereas for higher values of Ta, $N^{\text {sc }}$ decreases for lower values of $k$, and then increases for higher values of $k$.

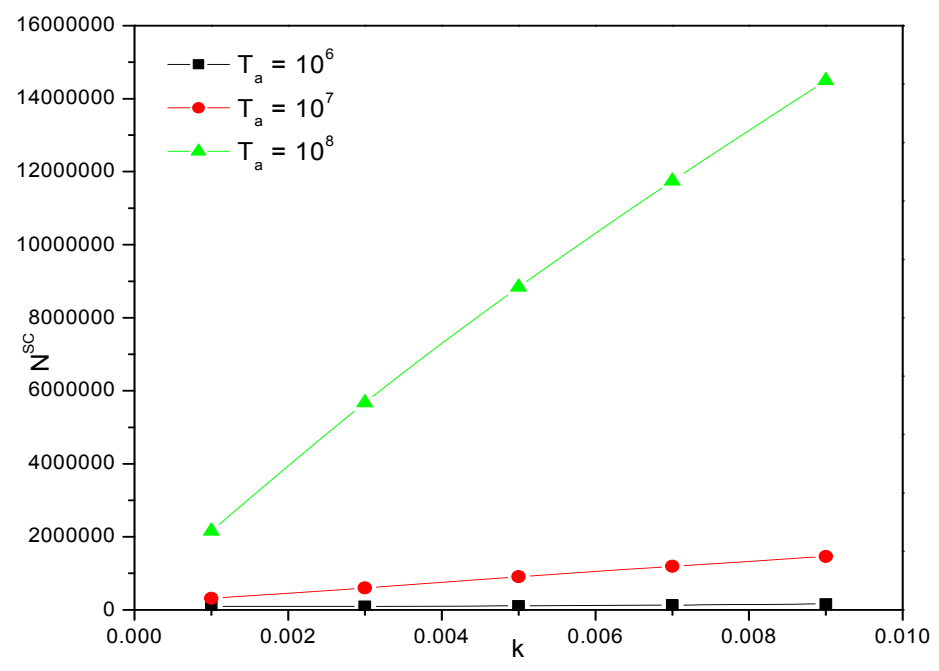

Fig.3. Variation of $N^{\mathrm{sc}}$ versus $k$ for different $\mathrm{Ta}$ with $\mathrm{St}=-0.002, R_{S}=-500, \tau=0.03$ and $M_{3}=5$. 
Figure 3 represents the variation of the thermal Rayleigh number $N^{\text {sc }}$ versus permeability of the porous medium $k$. It has been observed that as the Taylor number Ta is increased from $10^{6}$ to $10^{8}$, it is clear that as the permeability $k$ increases from 0.001 to 0.009 , the thermal Rayleigh number $N^{\mathrm{sc}}$ values increases. Therefore rotation leads to stability of the system.

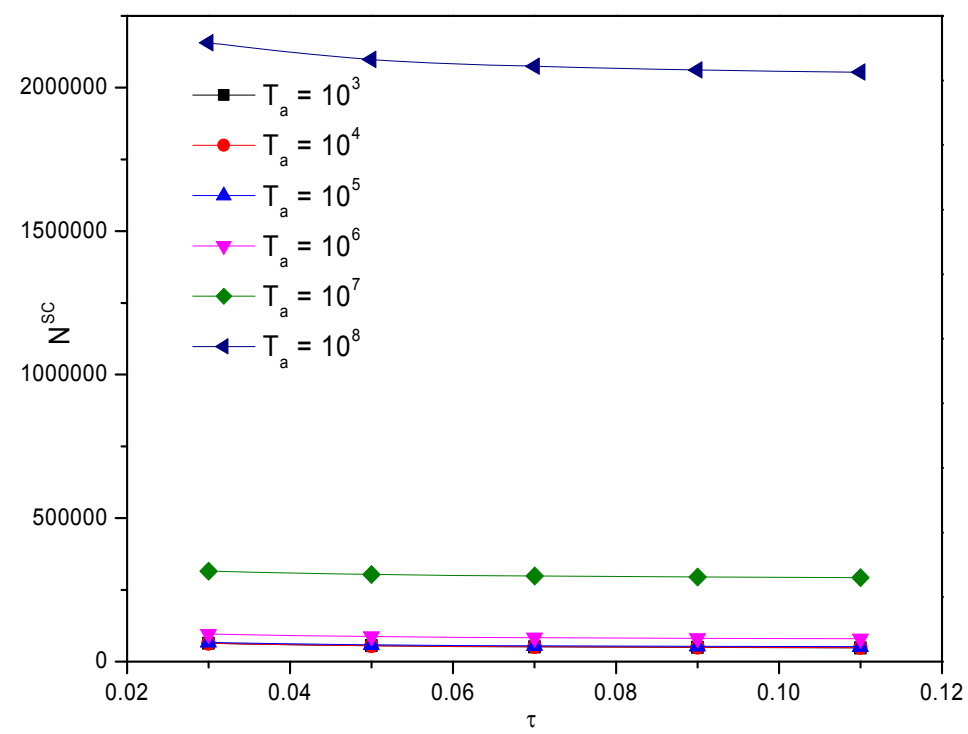

Fig.4. Variation of $N^{\mathrm{sc}}$ versus $\tau$ for different Ta with $\mathrm{St}=-0.002, R_{S}=-500, k=0.001$, and $M_{3}=5$.

Figure 4 gives the variation of the thermal Rayleigh number $N^{\mathrm{sc}}$ versus the ratio of mass transport to heat transport $\tau$ (varied from 0.03 to 0.11 ). It has been observed that as the Taylor number Ta is increased from $10^{3}$ to $10^{8}$, there is a notable variation in rotation. As the Taylor number Ta increases, the critical thermal Rayleigh number $N^{\mathrm{sc}}$ increases. Therefore, the effect of the Taylor number leads to stability of the system. It is clear that as the ratio of the mass transport to heat transport $\tau$ increases from 0.03 to 0.11 , the critical thermal Rayleigh number $N^{\text {sc }}$ is almost constant and the system is in equilibrium state due to rotation.

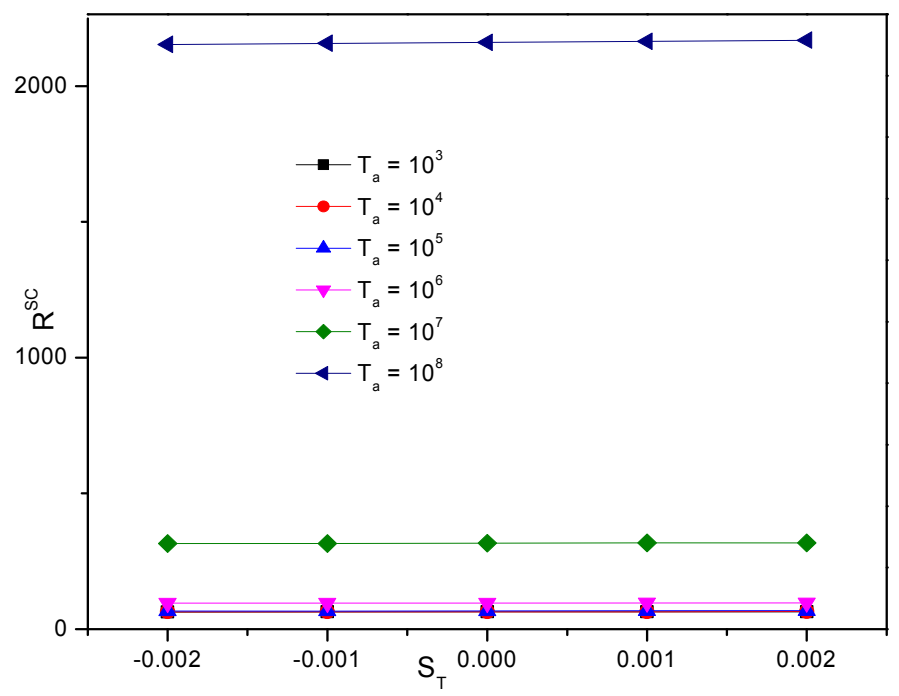

Fig.5. Variation of $R^{\mathrm{sc}}$ versus St for different Ta with $R_{S}=-500, k=0.001, \tau=0.03$ and $M_{3}=5$. 
Figure 5 indicates the variation of $R^{\text {sc }}$ versus St for different values of Ta. The figure shows a stabilizing behavior which is not much pronounced. The stabilization is minimal when the Taylor number Ta assumes values from $10^{3}$ to $10^{6}$, and then it increases phenomenally. This is indicated by an increase in $R^{\mathrm{sc}}$ values.

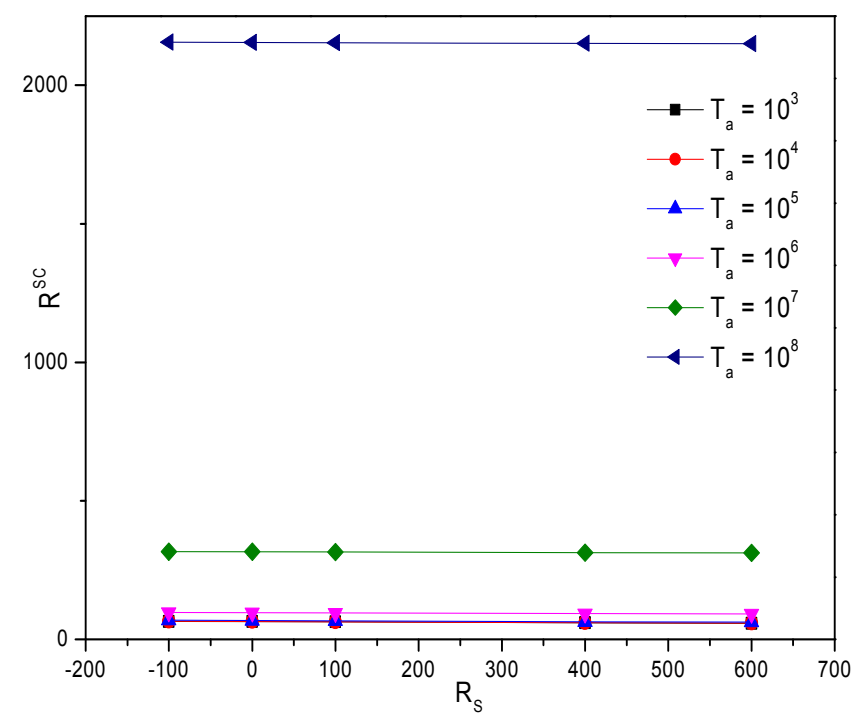

Fig.6. Variation of $R^{\mathrm{sc}}$ versus $R_{S}$ for different Ta with $\mathrm{St}=-0.002, k=0.001, \tau=0.03$ and $M_{3}=5$.

Figure 6 is a plot of the variation of $R^{\mathrm{sc}}$ versus $R_{\mathrm{S}}$ for different values of Ta. This figure shows that as Ta increases, there is an increase in the values of the critical magnetic Rayleigh number $R^{\mathrm{sc}}$. Therefore the Taylor number leads to stability of the system.

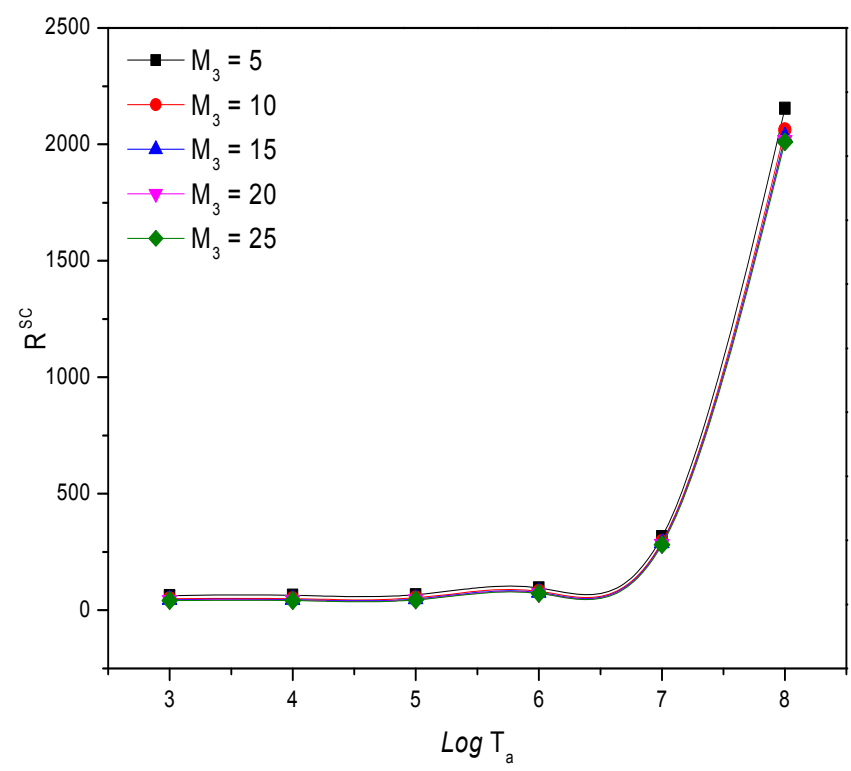

Fig.7. Variation of $R^{\mathrm{sc}}$ versus $\log \mathrm{Ta}$ for different $M_{3}$ with $R_{S}=100, k=0.001, \tau=0.03$ and $\mathrm{St}=-0.002$. 
Figure 7 analyzes the variation of the critical magnetic Rayleigh number $R^{\text {sc }}$ versus $\log$ Ta for various values of the non-buoyancy magnetization parameter $M_{3}$. When $\log$ Ta increases from 3 to 5 , stabilization is not much pronounced. But when it takes values from 6 to 8 , the increase in $R^{\text {sc }}$ values is maximum.

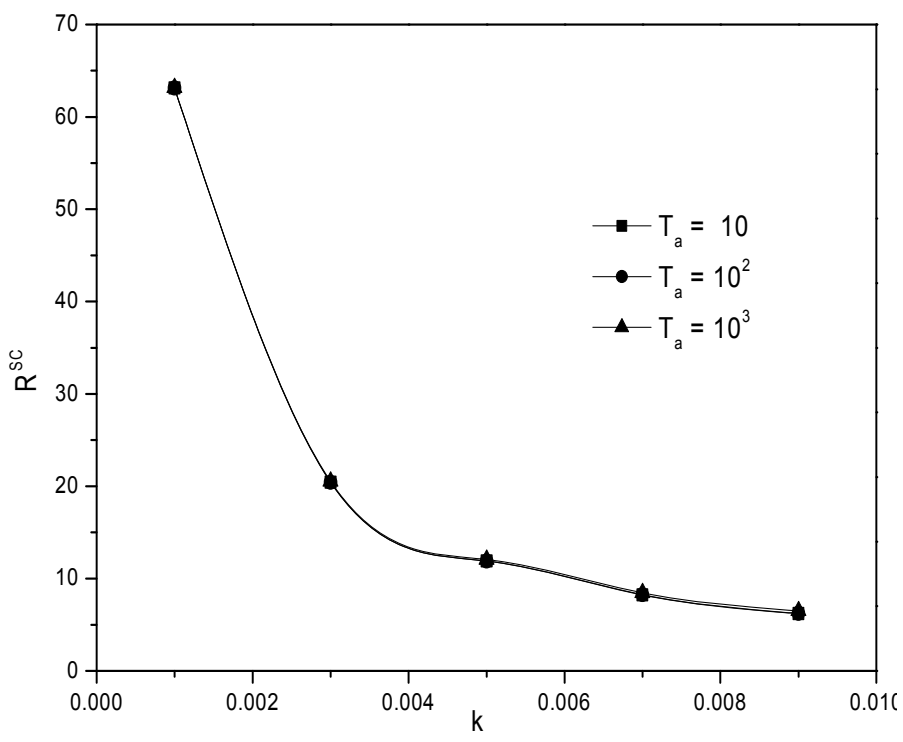

Fig.8. Variation of $R^{\mathrm{sc}}$ versus $k$ for different Ta with $\mathrm{St}=-0.002, R_{S}=100, \tau=0.03$ and $M_{3}=5$.

Figure 8 represents the variation of the critical magnetic Rayleigh number $R^{\text {sc }}$ versus permeability of the porous medium $k$ through stationary instability. It has been observed that as the Taylor number Ta is increased from 10 to $10^{3}$, there is no notable variation. The effect the Taylor number Ta is negligible. For different Ta, no appreciable changes in the curves are noticed. It is clear that as the permeability $k$ increases from 0.001 to 0.009 , the critical magnetic Rayleigh number $R^{\text {sc }}$ values tend to decrease leading to destabilization. This is due to the fact that an increase in the pore size makes the flow of the fluid easier causing instability to set in earlier.

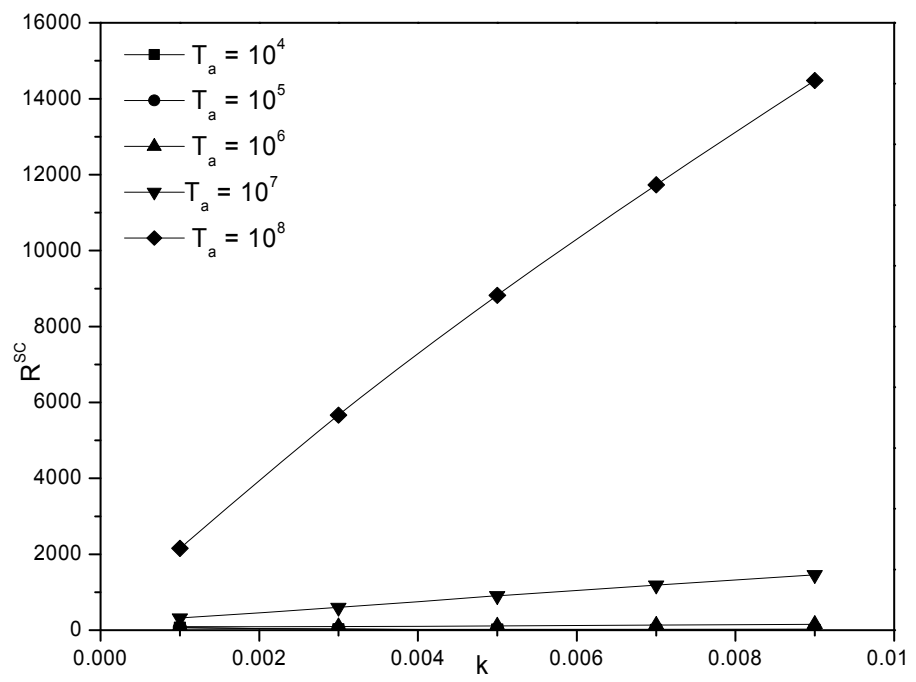

Fig.9. Variation of $R^{\mathrm{sc}}$ versus $k$ for different Ta with $\mathrm{St}=-0.002, R_{S}=100, \tau=0.03$ and $M_{3}=5$. 
Figure 9 gives the variation of the critical magnetic Rayleigh number $R^{\text {sc }}$ versus permeability of the porous medium $k$. It has been observed that as the Taylor number Ta is increased from $10^{4}$ to $10^{8}$, the critical magnetic Rayleigh number $R^{\mathrm{sc}}$ values tend to decrease leading to destabilization. But it is clear that as the permeability $k$ increases from 0.001 to 0.009 , the critical magnetic Rayleigh number $R^{\text {sc }}$ values tend to increase leading to stabilization.

\section{Conclusions}

The linear stability of thermohaline convection in a ferrofluid layer heated from below and salted from above saturating a densely packed porous medium subject to a transverse uniform magnetic field has been considered with the effect of rotation. In this investigation, the effect of various parameters such as permeability of the porous medium, non - buoyancy magnetization, buoyancy magnetization, Prandtl number, ratio of mass transport to heat transport, Rayleigh number, Taylor number and salinity Rayleigh number on the onset of convection has been calculated. The thermal critical magnetic Rayleigh numbers for the onset of instability are also determined numerically for sufficiently large values of the buoyancy magnetization parameter $M_{1}$ and results are depicted graphically. If $R^{\mathrm{oc}}>R^{\mathrm{sc}}$, then the system stabilizes through stationary mode. If $R^{\mathrm{oc}}<R^{\mathrm{sc}}$, then the system stabilizes through oscillatory mode, where $R^{\mathrm{oc}}$ and $R^{\mathrm{sc}}$ are the critical thermal Rayleigh numbers for the oscillatory and stationary convection system.

In conclusion, Figs 1 to 4 illustrate the critical thermal Rayleigh number $N^{\mathrm{sc}}$ versus $M_{3}, k$, and $\tau$ for different values of $\tau$ and Ta. But Figs 5 to 9 indicate the critical magnetic Rayleigh number $R^{\mathrm{sc}}$ versus $\mathrm{St}, R_{\mathrm{S}}$, $\log \mathrm{Ta}$, and $k$ for different values of $M_{3}$ and Ta. For the oscillatory convection, the Taylor number Ta has a stabilizing effect on various values of $k$ and $\tau$ which are studied in Figs $2-4$. But for various values of $M_{3}$, the convective system has a destabilizing effect which is analyzed in Fig.1. For stationary convection, the Taylor number Ta has a stabilizing effect for various values of St and $R_{\mathrm{S}}$ which are studied in Figs $5-6$. But for various values of $k$, the convective system has a destabilizing effect which is analyzed in Figs $8-9$. Figure 7 shows a stabilizing effect for Log Ta and a destabilizing effect for $M_{3}$, which are not much pronounced. Thus, rotation tends to stabilize the system.

Furthermore, the principle of exchange of instability is applied to find out the mode of attaining instability. It is found that the system stabilizes through stationary mode for values of $\mathrm{Ta} \leq 10^{3}$ and for oscillatory mode_instability is favored for $\mathrm{Ta}>10^{3}$. Thus convection is favoured in a ferromagnetic fluid by means of spatial variation in magnetization, which is induced when the magnetization of the ferrofluid depends on temperature and salinity.

\section{Nomenclature}

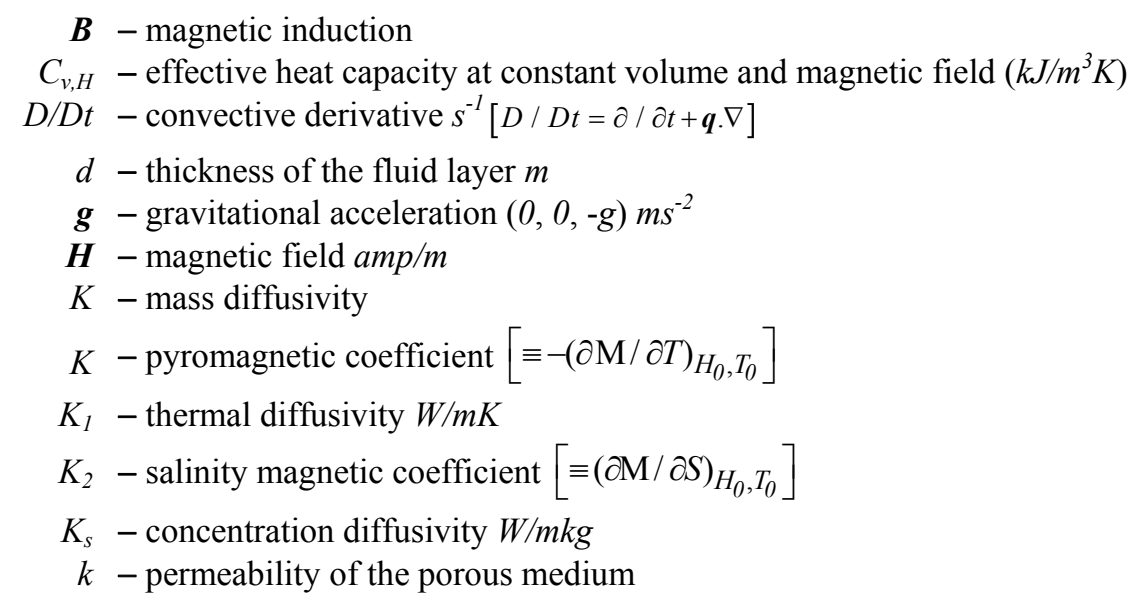




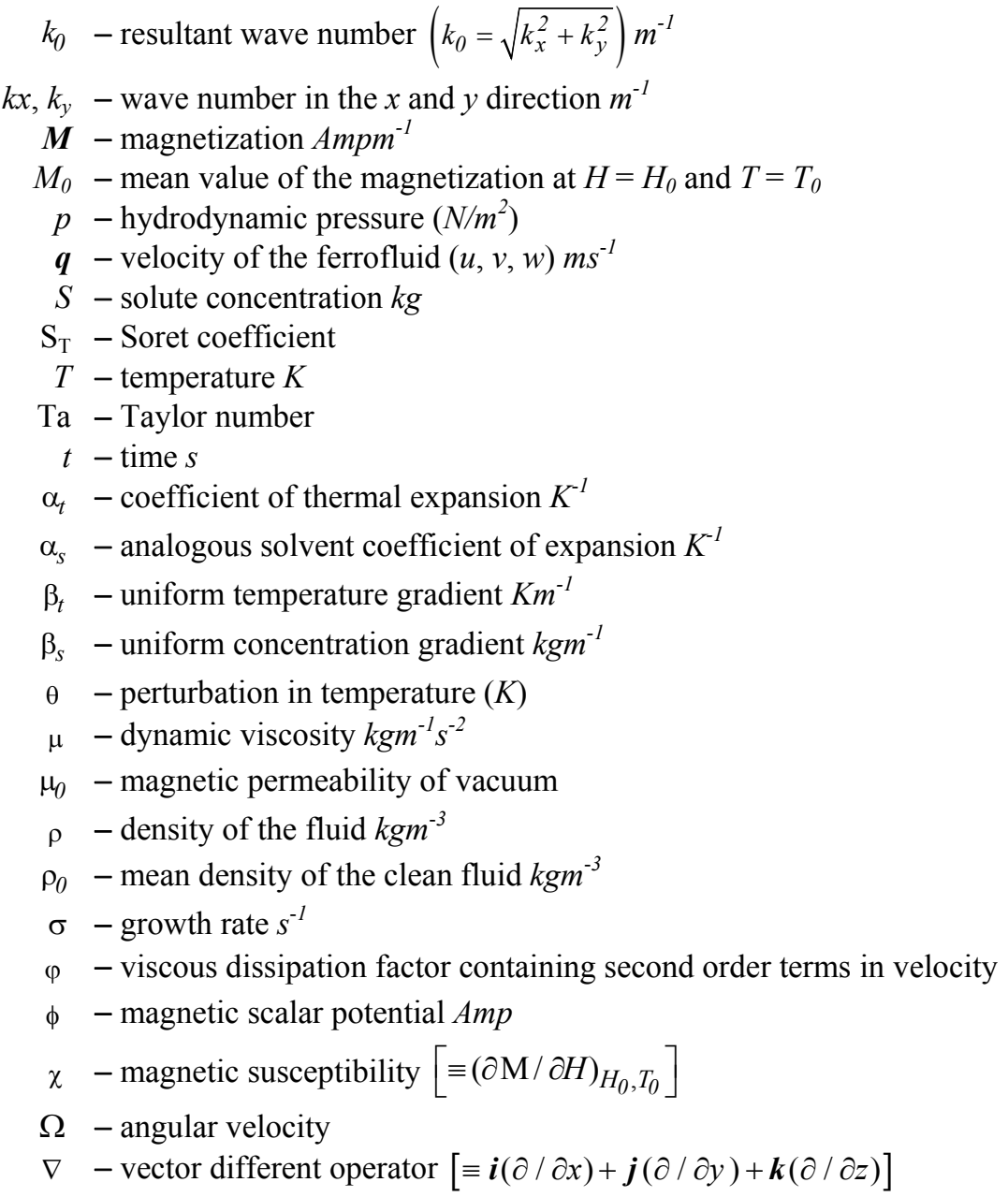

\section{References}

[1] Finlayson B.A. (1970): Convective instability of ferromagnetic fluids. - J. Fluid Mech., vol.40, pp.753-767.

[2] Das Gupta M. and Gupta A.S. (1979): Convective instability of a layer of a ferromagnetic fluid rotating about a vertical axis. - Int. J. Engng. Sci., vol.17, pp.271-277.

[3] Zhan M. and Rosensweig R.E. (1980): Stability of magnetiffluid penetration through a porous medium with uniform magnetic field oblique to the interface. - IEEE Transactions of Magnetic, vol.16, pp.275-282.

[4] Sekar R., Vaidyanathan G. and Ramanathan A. (1993): The ferroconvection in fluids saturating a rotating densely packed porous medium. - Int. J. of Engng. Sci., vol.31, pp.241-50.

[5] Sekar R. and Vaidyanathan G. (1993): Convective instability of a magnetized ferrofluid in a rotating porous medium. - Int. J. of Engng. Sci., vol.31, pp.1139-1150.

[6] Vaidyanathan G., Sekar R. and Ramanathan A. (1998): Effects of rotation and anisotropy of a porous medium on ferroconvection. - Ind. J. of Engng. and Mater. Sci., vol.5, pp.436-440.

[7] Sekar R., Vaidyanathan G. and Ramanathan A. (1998): Effects of rotation on ferrothermohaline convection saturating a porous medium. - Ind. J. Engng. and Mater. Sci., vol.5, pp.445-452.

[8] Sekar R., Vaidyanathan G. and Ramanathan A. (2000): Effect of rotation on ferrothermohaline convection. - J. of Magn. and Magn. Mater., vol.218, pp.266-272. 
[9] Hurle D.T.J. and Jakemann E.J. (1971): Soret-driven thermo solutal convection. - J. Fluid Mech., vol.47, pp.667687.

[10] Ryskin A., Muller H.W and Pleiner H. (2003): Thermo diffusion effects in convection of ferrofluids. Magnetohydrodynamics, vol.39, pp.51-55.

[11] Vaidyanathan G., Sekar R., Hemalatha R., Vasanthakumari R. and Senthilnathan S. (2005): Soret-driven ferrothermohaline convection. - J. of Magn. and Magn. Mater., vol.288, pp.460-469.

[12] Shevtsova M., Melnikov D.E. and Clegros J. (2006): Onset of convection in Soret driven instability. - Phys. Rev., E.73, pp.047302.

[13] Sekar R., Vaidyanathan G., Hemalatha R. and Senthilnathan S. (2007): Effect of Coriolis force on a Soret driven ferrothermohaline convective system. - Int. J. of Math. Sci., vol.6 pp.429-441.

[14] Vaidyanathan G., Sekar R. and Hemalatha R. (2007): Effect of coriolis force on a soret driven ferrothermohaline convection in a medium of sparse particle suspension. - Ind. J. of Pure and Appl. Phys., vol.45, pp.666-673.

[15] Sunil, Chand P., Mahajan A. and Sharma P. (2011): Effect of rotation on double diffusive convection in a magnetized ferrofluid with internal angular momentum. - J. Appl. Fluid Mech., vol.4, No.4, pp.43-52.

[16] Shivakumara I.S., Sureshkumar S. and Devaraju N. (2011): Coriolis effect on thermal convection in a couple stress fluid saturated rotating rigid porous layer. - Arch. of Appl. Mech., vol.81, pp.513-530.

[17] Ram P., Kumar V. (2012): Ferrofluid flow with magnetic field dependent viscosity due to rotating disk in porous medium. - Int. J. Appl. Mech., vol.4, No.4, pp.1250041.

[18] Ram P. and Sharma K. (2014): Effect of rotation and MFD viscosity on ferrofluid flow with rotating disk. - Ind. J. Pure and Appl. Phy., vol.52, pp.87-92.

[19] Sekar R., Vaidyanathan G., Hemalatha R. and Senthilnathan S. (2006): Effect of sparse distribution pores in a Soret-driven ferro thermohaline convection. - J. of Magn. and Magn. Mater., vol.302, pp.20-28.

[20] Sekar R., Raju K. and Vasanthakumari R. (2013): A linear analytical study on Soret-driven ferrothermohaline convection in an anisotropic porous medium. - J. of Magn. and Magn. Mater., vol.331, pp.122-128.

[21] Sekar R., Murugan D. and Raju K. (2013a): Stability analysis of thermohaline convection in ferromagnetic fluid in densely packed porous medium with Soret effect. - World J. of Engng., vol.10, pp.439-447.

[22] Sekar R. and Raju K. (2013): Effect of magnetic field dependent viscosity on Soret-driven thermoconvective instability of ferromagnetic fluid in the presence of rotating anisotropic porous medium of sparse particle suspension. - Int. J. of Math. Sci., vol.12, pp.13-31.

[23] Sekar R. and Raju K. (2014): Effect of magnetic field dependent viscosity on Soret-driven ferrothermohaline convection saturating an anisotropic porous medium of sparse particle suspension. - World J. of Engng., vol.11, pp.213-228.

[24] Sekar R. and Raju K. (2014a): Effect of Soret and temperature dependent viscosity on thermohaline convection in a ferrofluid saturating a porous medium. - Int. J. of Appl. Mech. and Engng., vol.19, pp.321-336.

[25] Sekar R., Raju K. and Vasanthakumari R. (2013b): Linear stability analysis of coriolis force on ferrothermohaline convection saturating an anisotropic porous medium with Soret effect. - Global J. of Math. Analy., vol.1, pp.3747.

[26] Sekar R., Murugan D. and Raju K. (2016): Densely distribution pores and Coriolis force on thermohaline convection in a ferrofluid with Soret and anisotropy effects. - Int. J. Advanced Sci. and Tech., vol.87, pp.15-30.

[27] Sekar R., Murugan D. and Raju K. (2016a): Ferrothermoconvective instability in Soret driven convection saturating a densely packed anisotropic porous medium. - Int. J. Appl. Math. Electronics and Computers vol.4, No.2, pp.58-64.

Received: March 28, 2018

Revised: June 12, 2018 